\title{
Breaking the 100 MG Barrier: The First High Field Magnetic CV
}

\author{
Paula Szkody \\ Dept. of Astronomy, University of Washington, Box 351580, Seattle, \\ WA 98195
}

Gary D. Schmidt and Paul S. Smith

Steward Observatory, University of Arizona, Tucson, AZ 85721

Andrew Silber and D.W. Hoard

Dept. of Astronomy, University of Washington, Box 351580, Seattle, WA 98195

Gaghik Tovmassian

Instituto de Astronomia, UNAM, Apdo. Post 877, 22860, Ensenada, B.C., Mexico

B.T. Gänsicke

Universitat-Sternwarte, Geismarlandstrasse 11, D-37083, Gottingen, Germany

D. de Martino

IUE Observatory, VILSPA-ESA, P.O. Box 50727, E-28080 Madrid, Spain

\begin{abstract}
We present results from IUE and optical spectra, optical photometry and circular polarimetry during high and low states of the highly luminous soft X-ray cataclysmic variable AR UMa that identifies the primary in this system as a white dwarf with a magnetic field of 230 MG. The high magnetic field likely threads accretion blobs all the way from the secondary to below the surface of the white dwarf, resulting in a lack of polarised cyclotron emission and an extreme soft-X-ray luminosity during the high state.
\end{abstract}

\section{Introduction}

The cataclysmic variable AR UMa was first identified as the optical counterpart of an extremely variable, extremely soft Einstein source (Remillard et al. 1994). Their search through the Harvard plate files showed there were high and low states varying between 14 and 16.5 magnitude. They obtained optical spectra and I-band photometry during a low state, which revealed TiO bands from an 
M6 dwarf (giving a distance of $88 \mathrm{pc}$ ) and an ellipsoidal variation from this dwarf which gave a binary orbital period of $1.932 \mathrm{hr}$. On the basis of these traits, they suggested that AR UMa was likely an AM Her type of system, in which a magnetic (10-70 MG field) white dwarf dominates the accretion flow and prevents the formation of an accretion disk (Cropper 1990).

\section{Observations}

Our observations encompassed IUE and optical spectra, as well as broad band photometry and circular polarimetry during the high state from April to October 1995. This was followed by low state observations from November 1995 to March 1996 that included the above kinds of data as well as time-resolved spectropolarimetry. The IUE data provided spectra in the short wavelength camera at low dispersion. The high state spectra were obtained with the $3.5 \mathrm{~m}$ telescope at a resolution of $2 \AA$. The high state photometry was accomplished with a CCD on the $1.5 \mathrm{~m}$ telescope at OAN de San Pedro Martir, while the polarimetry and spectropolarimetry during both states was accomplished with the 1.5 and $2.3 \mathrm{~m}$ telescopes at Steward Observatory.

\section{Discussion}

The high state state optical spectra show strong optical emission lines, with the HeII 4686 emission stronger than $\mathrm{H} \beta$ and a continuum slope $F_{\lambda} \propto \lambda^{-2.0}$. The IUE spectrum also shows strong UV emission lines, with a weak UV continuum. The four nights of optical photometry folded on the orbital period reveal a modulated light curve reminiscent of magnetic systems like AM Her and EF Eri, which have one pole continuously in view (Cropper 1990). The most surprising aspect of the high state data is that only a very marginal circular polarization (V/I 0.4\%) was detected.

In contrast, the low state broad band polarization reveals a sinusoidal variation from +2.2 to $+4.3 \%$ during the orbital period. The optical spectra show narrow Balmer emission lines, with no Hell emission, and a steep $\left(F_{\lambda} \propto \lambda^{-3.8}\right)$ blue continuum. The emission line strengths decrease from Nov. 1995 to Jan. 1996. The optical spectropolarimetry (Figure 1) reveals strongly polarized dips which resemble photospheric Zeeman lines from a magnetic white dwarf, but the features do not correspond to known patterns from hydrogen or helium. The IUE continuum at the low state is very similar in flux to the high state, but the emission lines have disappeared and the only evident feature is broad absorption near $1300 \AA$, which is consistent with a Zeeman component of Ly $\alpha$ in a dipole field of $230 \mathrm{MG}$ (Figure 1). In these respects, AR UMa resembles the isolated magentic white dwarfs GD229, LB11146b and PG1031+234 (Schmidt et al. 1996a; Liebert et al. 1993; Schmidt et al. 1986). The radial velocity curve and near IR light curve constructed from the optical spectrapolarimetry in March 1996 show that the line emission at the low state originates from the irradiated secondary, while the circular polarization combined with the radial velocities reveals that the assumed dipole axis of the field pattern is oriented at $90^{\circ}$ to the line of centers. 

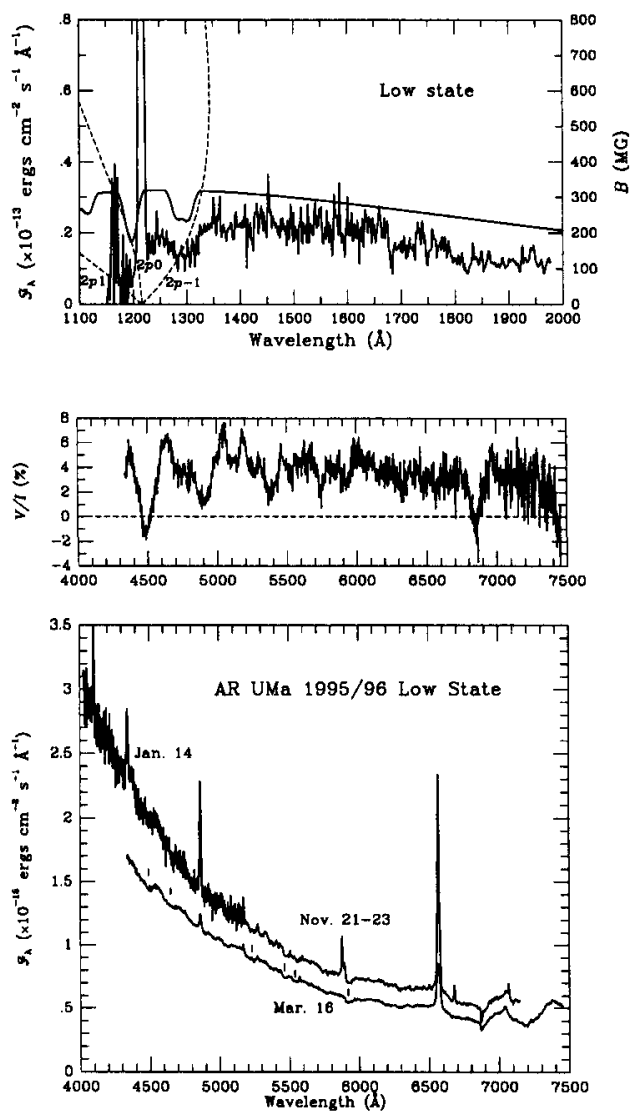

Figure 1. The low state IUE spectrum compared with a photospheric model for Ly $\alpha$ in a dipolar magnetic field of $230 \mathrm{MG}$ (top); the circular polarization spectrum in March 1996 coadded over the orbit (middle); the optical spectra during the low state (bottom) with the unidentified polarized absorption features marked in the March spectrum. 


\section{Conclusions}

The previous difficulties in identifying high field AM Her stars can be better understood from the known properties of AR UMa. The pole-on orientation plus the large field strength resulting in the cyclotron fundamental occurring in the optical results in low polarization during high states of mass accretion. The strong magnetic field likely threads blobs all the way to the secondary, resulting in clumps of material burying deep into the white dwarf, producing the observed soft $\mathrm{X}$-ray excess. The long times spent at low states result in selection effects against discovery. This means that the best way to find other high field systems is probably to look for soft X-ray objects during low states. Further details will be available in Schmidt et al. (1996b).

Acknowledgments. This work was partially supported by NASA grants NAGW-3158 and NSF grant AST 92-17911 to PS and AST 91-14087 to GDS.

\section{References}

Cropper, M. 1990, Sp. Sci. Rev., 54, 195

Liebert, J., Bergeron, P., Schmidt, G.D., \& Saffer, R.A. 1993, ApJ, 418, 426

Remillard, R.A., Schachter, J. F., Silber, A.D. \& Slane, P. 1994, ApJ, 426, 288

Schmidt, G.D., Stockman, H.S., \& Grandi, S.A. 1986, ApJ, 300, 804

Schmidt, G.D., Allen, R., Smith, P., \& Liebert, J. 1996a, ApJ, in press

Schmidt, G.D., Szkody, P., Smith, P., Silber, A., Tovmassian, G., Hoard, D.W., Gänsicke, B.T. \& de Martino, D. 1996b, ApJ, in press

\section{Discussion}

M. Livio: It will be interesting to attempt to determine the white dwarf mass. This could both have implications for the theoretical model and for comparison with field white dwarfs, where the high field white dwarfs seem perhaps to be on the massive side.

P. Szkody: Yes, this will be an important point to pursue with further UV and optical time-resolved data. 